\title{
Bilateral Acute Retinal Necrosis and Encephalomalacia Due to Herpes Simplex Virus Infection in a Premature Infant
}

Mi Hye Bae ${ }^{1}$, Na Rae Lee ${ }^{2}$, Young Mi Han², Lira Yoon ${ }^{1}$, Young Mi Kim', Shin Yun Byun², Jae Jung Lee ${ }^{3}$, Han Jo Kwon $^{4}$, Min Jung Kwak ${ }^{1}$, and Kyung Hee Park ${ }^{1}$

${ }^{1}$ Department of Pediatrics, Pusan National University Hospital, Pusan National University School of Medicine and Biomedical Research Institute, Busan, Korea

${ }^{2}$ Department of Pediatrics, Pusan National University Children's Hospital, Pusan National University School of Medicine, Yangsan, Korea ${ }^{3}$ Department of Ophthalmology, Pusan National University School of Medicine, Yangsan, Korea

${ }^{4}$ Biomedical Research Institute, Pusan National University Hospital, Busan, Korea

\section{ABSTRACT}

Herpes simplex virus (HSV) is a common pathogen, that causes a broad spectrum of diseases, ranging from minor skin infections to severe encephalitis and widespread infections. Acute retinal necrosis (ARN), one of the most serious manifestations of HSV infection, is defined as a rapidly progressing necrotizing retinopathy that presents discrete areas of circumferential retinal necrosis, along with signs of uveitis, vitreitis, and retinal vasculitis. We encountered a case of a female infant, born at 33 weeks of gestation with a body weight at birth of 2,080 g, who had ARN and encephalomalacia due to HSV infection. ARN associated with HSV infection should be suspected when nonspecific retinal exudates are observed in neonates, especially preterm infants.

Key Words: Herpesvirus 2, human; Retinal necrosis syndrome, acute; Infant, premature

\section{INTRODUCTION}

Herpes simplex virus (HSV) infection in neonates is associated with considerable mortality if not recognized and treated early. Especially, encephalitis due to HSV infection in newborns has high rates of morbidity and mortality ${ }^{1)}$.

Acute retinal necrosis (ARN), one of the most serious manifestations of HSV infection, is defined as a rapidly progressing necrotizing retinopathy that presents discrete areas of circumferential retinal necrosis, along with signs of uveitis, vitreitis, and retinal vasculitis ${ }^{2}$. The potential complications of ARN include ischemic optic neuropathy and exudative retinal detachment that can lead to severe vision loss.

To our best knowledge, this is the first report of a case of combined ARN and encephalo-
Received: 10 August 2018

Revised: 26 October 2018

Accepted: 29 November 2018

Correspondence to: Kyung Hee Park

Departments of Pediatrics, Pusan National University Hospital, Pusan National University School of Medicine, 179 Gudeok-ro, Seo-gu, Busan 49241, Korea

Tel: +82-51-240-7293

Fax: +82-51-248-6205

E-mail: kuma2002@hanmail.net http://orcid.org/0000-0002-1028-4225

Copyright(c)

By Korean Society of Neonatology.

All right reserved.

This is an Open-Access article distributed under the terms of the Creative Commons Attribution Non-Commercial License (http://creativecommons.org/licenses/ by-nc/4.0), which permits unrestricted non-commercial use, distribution, and reproduction in any medium, provided the original work is properly cited. 
malacia due to HSV infection in a Korean patient. Herein, we described a case of a female baby, born at a gestational age (GA) of 33 weeks, who developed ARN and encephalomalacia due to HSV type 2.

\section{CASE REPORT}

A female infant was referred to our neonatal intensive care unit (NICU) at 41 weeks GA for the evaluation of nonspecific retinal exudates and worsened apnea. The patient's first hospitalization was due to prematurity. She was born at 33 weeks of GA, weighing $2,080 \mathrm{~g}$, through normal vaginal delivery. The Apgar scores were 7 at 1 minutes and 9 at 5 minutes after delivery. The postnatal period was uneventful, and the patient was discharged at a postmenstrual age (PMA) of 38 weeks. At 37 weeks PMA, the first ophthalmic examination was performed for retinopathy of prematurity (ROP), which showed the presence of wedge shaped retinal exudates. She was referred to other ophthalmologists, but they could not determine the cause of the retinal exudates. At 40 weeks PMA, she was re-admitted because of the development of apnea and poor sucking. During the second admission, her symptoms were worsened and she was referred to our hospital's NICU at 41 weeks PMA.

Physical examination revealed mental stupor and poor responsiveness. The pupils were constricted but reactive to light. Tests for sepsis and metabolic disease were completed. The blood chemistry results and electrolyte levels were within the normal ranges. The cerebrospinal fluid (CSF) test revealed pleocytosis (92 lymphocytes/ $\mu \mathrm{L}$ ), increased protein levels $(600 \mathrm{mg} / \mathrm{dL})$, and decreased glucose level (CSF to serum glucose ratio, 40\%). CSF culture and meningitis/encephalitis polymerase chain reaction (PCR) panel were sent for evaluation. We diagnosed meningoencephalitis based on the patient's presenting symptoms and CSF results, and initiated treatment with empirical antibiotics of (cefotaxime and ampicillin). However, her desaturation and apnea worsened. She was intubated and placed on synchronized intermittent mechanical ventilation. On hospital day 2, the HSV type 2 PCR of the CSF yielded a positive result. Electroencephalography showed markedly abnormal results due to severe suppression of background activities. Brain magnetic resonance imaging revealed encephalomalacia at both cerebral hemispheres and the corticospinal tract (Figure 1).

Dilated fundus examination was performed to evaluate the retinal exudates and revealed multiple focal exudative retinal detachments with retinal whitening in both eyes, consistent with ARN (Figure 2). Encephalomalacia and ARN due to HSV infection were diagnosed and the infant was administered with acyclovir $20 \mathrm{mg} / \mathrm{kg}^{-1} /$ day $^{-1}$ intravenously for 3 weeks.

Serologic tests for toxoplasma, syphilis, rubella, and cytomegalovirus were performed and produced unremarkable results. Serum HSV serology detected HSV-2 immunoglobulin M (IgM) and IgG. Moreover, we conducted a thorough review of the parents' history. The mother was 37 years old, gravida 4, para 3, when the infant was born. No relevant events occurred during the pregnancy, and there were no maternal complications associated with the pregnancy. The mother had not reported any history of genital HSV infection during pregnancy or at the time of delivery.

We attempted to wean the infant from mechanical ventilation
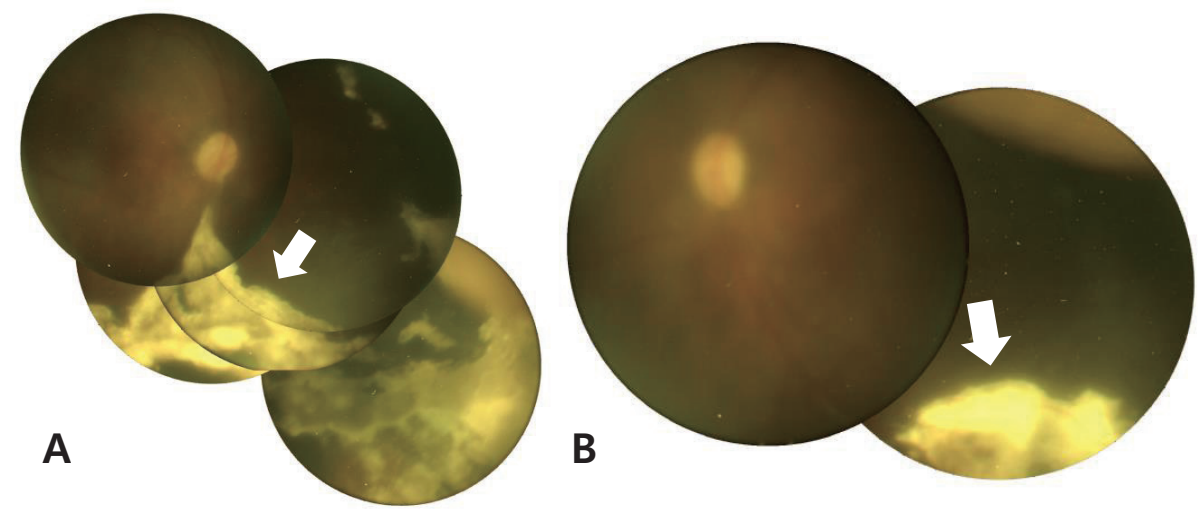

Figure 1. Fundus photographs showing exudative lesions such as retinitis (white arrows), vitreous haziness, pale disc, and peripheral atrophic lesions of the (A) right eye and (B) left eye. 
several times, but failed. Finally, she was discharged with a tracheostomy and an indication of home ventilation at 3 months of age. Currently, at age 5 months, she has severe neurodevelop mental delay and is expected to have poor visual outcome. Written informed consent was obtained from both parents.

\section{DISCUSSION}

HSV is a common pathogen, that causes a broad spectrum of diseases, ranging from minor skin infections to severe encephalitis and widespread infections. Most of the affected neonates acquire the infection from the maternal genital tract during delivery $(86 \%)^{3)}$. However, $60 \%$ to $80 \%$ of the mothers have no medical history of contact with a partner with genital HSV and present no signs or symptoms of genital herpes during delivery ${ }^{2)}$. Neonatal HSV infection is associated with considerable neurologic morbidity and complication due to seeding of asymptomatic virus and underdiagnosis of the condition ${ }^{4)}$.

ARN has been occasionally reported in the pediatric population ${ }^{5,6)}$. Most pediatric cases of ARN are caused by reactivation of a previous infection in immunocompetent or compromised patients ${ }^{7)}$. HSV type 2 is the most common cause of ARN in child$\operatorname{hood}^{8-10)}$. However, most ARN cases are known to occur in adults aged 20 to 60 years because of reactivation of a previous viral meningitis or encephalitis infection, with reported latencies of up to 46 years $^{8,11-13)}$.
In contrast to pediatric or adult cases, neonatal case series postulate that HSV-mediated ARN is a primary infection rather than a viral reactivation because neonates would not yet have acquired immunity to HSV ${ }^{14,15)}$. There has been much focus on HSV encephalitis in neonatal HSV infection. Nevertheless, ocular manifestations and retinal evaluation of these infants have been rarely reported, and the management of neonatal ocular lesions is also less often reported. In fact, ARN of neonates or infants has been rarely reported ${ }^{1)}$. Most neonatal ARN cases involve preterm infants who simultaneously show symptoms due to HSV encephalopathy ${ }^{2,14,16)}$.

It is not easy to suspect ARN when the patient is a preterm infant with retinal exudates, that were incidentally found on ROP examination. Unfortunately, nonspecific retinal exudates could not lead to the diagnosis of ARN when the first ophthalmic examination was done at 37 weeks PMA, as in our patient. Retinal exudate in neonates is not a common symptom. However, Fong et al. ${ }^{14)}$ reported a case of neonatal ARN, in which nonspecific exudate was found in the posterior segment at the first ophthalmic assessment, although the causative virus was HSV type 1. Therefore, this case highlights the need to suspect ARN and HSV infection when ophthalmic examination reveals retinal exudates in neonates, especially preterm infants.

Neonates, especially those born prematurely, may be at an increased risk for HSV-2 ARN secondary to a relatively immunocompromised state. Venincasa et al. ${ }^{16)}$ reported three cases of neonatal ARN. Among the three neonates, two were born pre-

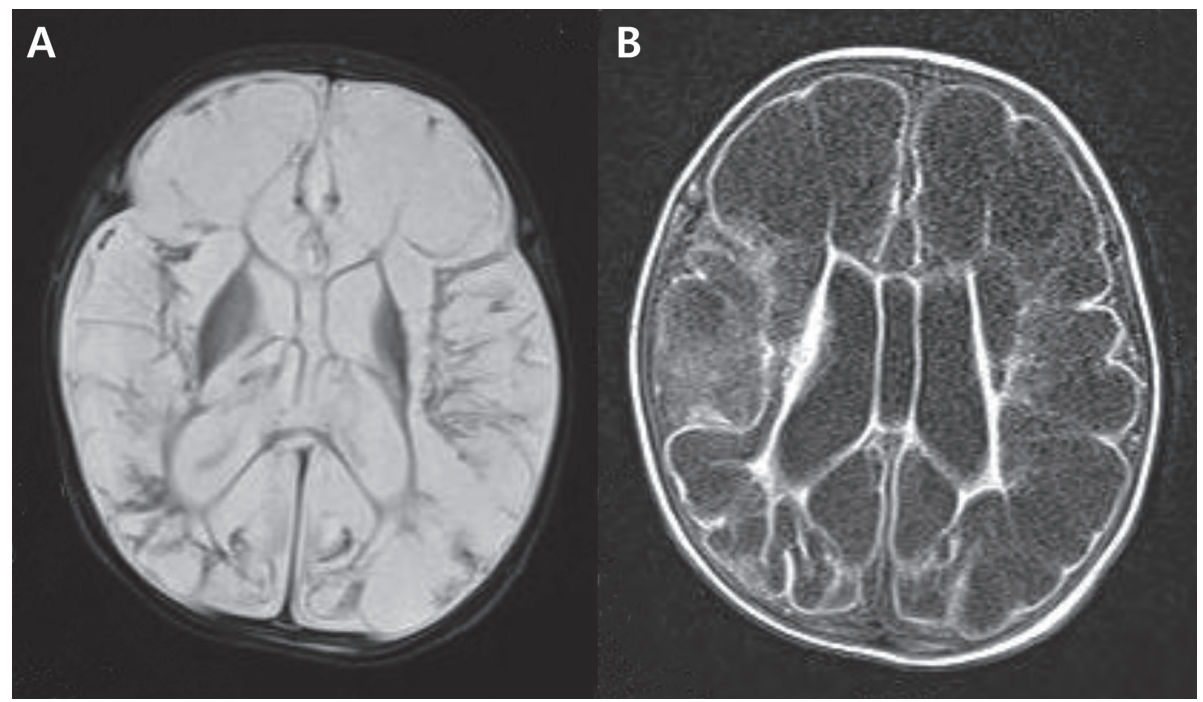

Figure 2. Brain magnetic resonance imaging showed encephalomalacia at both cerebral hemispheres and the corticospinal tract, consistent with herpes simplex virus encephalitis, in the (A) T2-weighted image and (B) fluid-attenuated inversion recovery image. 
maturely at 32 and 29 weeks GA, respectively. The first neonate was a 2-month-old female infant delivered at 32 weeks GA who had seizures and diffuse encephalopathy. Dilated fundus examination showed dense vitritis and a total exudative retinal detachment with peripheral whitening in the right eye, consistent with ARN. PCR analysis of aqueous fluid was positive for HSV.

Wong et al. ${ }^{15)}$ reported a case of an infant born at 30 weeks GA who, at 37 weeks PMA, presented with bilateral ARN and HSV encephalomalacia. This case was very similar to our present case. However, in contrast to our patient, their baby had a good ophthalmologic outcome owing to early diagnosis.

As the neonatal condition can deteriorate quickly, an imme diate and accurate diagnosis is desirable. Delayed diagnosis and treatment might cause poor ophthalmic and severe neurodevelopmental sequelae, as in our patient. Ophthalmic symptoms such as nonspecific retinal exudates appeared before other systemic symptoms, including worsened apnea, in our patient. Therefore, ARN associated with HSV infection should be suspected when nonspecific retinal exudates are observed in neonates, especially preterm infants.

\section{CONFLICT OF INTEREST}

No potential conflict of interest relevant to this article was reported.

\section{ACKNOWLEDGMENTS}

This work was supported by a clinical research grant from Pusan National University Hospital 2017.

\section{REFERENCES}

1. Basak RB, Malpani V, Kakish K, Vargese S, Chauhan N, BoeckA. Poor neurological sequelae of herpes simplex virus encephalitis in an infant despite adequate antiviral and adjunct corticosteroid therapy. Indian J Dermatol 2011;56:749-51.
2. Hsu C, Moinfar N, Lipman B, Capone A, Trese M. Acute retinal necrosis in a neonate. Retin Cases Brief Rep 2013;7:406-8.

3. Pinninti SG, Kimberlin DW. Neonatal herpes simplex virus in fections. Pediatr Clin North Am 2013;60:351-65.

4. Cherpes TL, Matthews DB, Maryak SA. Neonatal herpes simplex virus infection. Clin Obstet Gynecol 2012;55:938-44.

5. Kychenthal A, Coombes A, Greenwood J, Pavesio C, Aylward GW. Bilateral acute retinal necrosis and herpes simplex type 2 encephalitis in a neonate. Br J Ophthalmol 2001;85:629-30.

6. Moesen I, Khemka S, Ayliffe W. Acute retinal necrosis secondary to herpes simplex virus type 2 with preexisting chorioretinal scarring. J Pediatr Ophthalmol Strabismus 2008;45:59-61.

7. Thompson WS, Culbertson WW, Smiddy WE, Robertson JE, Rosenbaum JT. Acute retinal necrosis caused by reactivation of herpes simplex virus type 2. Am J Ophthalmol 1994;118:205-11.

8. Ganatra JB, Chandler D, Santos C, Kuppermann B, Margolis TP. Viral causes of the acute retinal necrosis syndrome. Am J Ophthalmol 2000;129:166-72.

9. Van Gelder RN, Willig JL, Holland GN, Kaplan HJ. Herpes simplex virus type 2 as a cause of acute retinal necrosis syndrome in young patients. Ophthalmology 2001;108:869-76.

10. Tan JCH, Byles D, Stanford MR, Frith PA, Graham EM. Acute retinal necrosis in children caused by herpes simplex virus. Retina 2001;21:344-7.

11. Silva RA, Berrocal AM, Moshfeghi DM, Blumenkranz MS, Sanislo S, Davis JL. Herpes simplex virus type 2 mediated acute retinal necrosis in a pediatric population: case series and review. Graefes Arch Clin Exp Ophthalmol 2013;251:559-66.

12. Lau CH, Missotten T, Salzmann J, Lightman SL. Acute retinal necrosis features, management, and outcomes. Ophthalmology 2007;114:756-62.

13. La Cava M, Abbouda A, Restivo L, Zito R. Delayed onset of bilateral acute retinal necrosis syndrome: a 46-year interval. Semin Ophthalmol 2015;30:146-9.

14. Fong CY, Aye AM, Peyman M, Nor NK, Visvaraja S, Tajunisah I, et al. Neonatal herpes simplex virus type-1 central nervous system disease with acute retinal necrosis. Pediatr Infect Dis J 2014;33:424-6.

15. Wong RK, Khanifar AA, Sun G, Heier LA, Saffra N, Chan RV. Acute retinal necrosis and cystic encephalomalacia in a premature neonate. Retin Cases Brief Rep 2010;4:202-5.

16. Venincasa VD, Emanuelli A, Leng T, Perlini E, Villegas V, DiazBarbosa M, et al. Acute retinal necrosis secondary to herpes simplex virus type 2 in neonates. Ophthalmic Surg Lasers Imaging Retina 2015;46:499-501. 